\title{
A relativistic dissipative hydrodynamic description for systems including particle number changing processes
}

\author{
Andrej $\mathrm{El}^{1 *}$, Azwinndini Muronga ${ }^{2 \dagger}$, Zhe $\mathrm{Xu}^{1,3 \ddagger}$, Carsten Greiner ${ }^{1 \S}$ \\ 1 Institut für Theoretische Physik, Goethe-Universität Frankfurt, \\ Max-von-Laue Strasse 1, D-60438, Frankfurt am Main, Germany \\ 2 Institute for Theoretical Physics and Astrophysics, \\ Department of Physics, University of Cape Town, Rondebosch 7701, \\ South Africa; UCT-CERN Research Centre, Department of Physics, \\ University of Cape Town, Rondebosch 7701, South Africa and \\ 3 Frankfurt Institute for Advanced Studies, \\ Ruth-Moufang-Strasse 1, D-60438, Frankfurt am Main, Germany
}

\begin{abstract}
Relativistic dissipative hydrodynamic equations are extended by taking into account particle number changing processes in a gluon system, which expands in one dimension boost-invariantly. Chemical equilibration is treated by a rate equation for the particle number density based on Boltzmann equation and Grad's ansatz for the off-equilibrium particle phase space distribution. We find that not only the particle production, but also the temperature and the momentum spectra of the gluon system, obtained from the hydrodynamic calculations, are sensitive to the rates of particle number changing processes. Comparisons of the hydrodynamic calculations with the transport ones employing the parton cascade BAMPS show the inaccuracy of the rate equation at large shear viscosity to entropy density ratio. To improve the rate equation, the Grad's ansatz has to be modified beyond the second moments in momentum.
\end{abstract}

PACS numbers: 47.75.+f, 24.10.Lx, 24.10.Nz, 12.38.Mh, 25.75.-q

\footnotetext{
* el@th.physik.uni-frankfurt.de

$\dagger$ Azwinndini.Muronga@uct.ac.za

$\ddagger$ xu@th.physik.uni-frankfurt.de

$\S$ Carsten.Greiner@th.physik.uni-frankfurt.de
} 


\section{INTRODUCTION}

Relativistic dissipative hydrodynamics has established itself as an effective theory for investigations of phenomena in relativistic heavy-ion collisions owing to its success in describing the elliptic flow $v_{2}$ data measured at the Relativistic Heavy Ion Collider (RHIC) at Brookhaven National Laboratory (BNL) [1-6]. Most hydrodynamic approaches [2 4, 7, 8] do assume an instantaneous chemical equilibrium of matter constituents. However, this requires an infinite large transition rate of microscopic processes which drive the system towards the chemical equilibrium and thus breaks the consistency of the physical description for matter with a finite viscosity. Another extreme but physically consistent case, as considered in

Ref. [9], is to keep the number of the constituents constant by assuming that there are no particle number changing processes. In this case deviation of the one-particle phase space distribution from its chemical equilibrium form becomes larger and larger when the system expands. In real ultrarelativistic heavy-ion collisions bremsstrahlung processes of gluons and quarks, also gluon fusion to quark and antiquark, and the reverse processes govern the chemical equilibration of gluons and quarks [10-14]. Therefore, particle number changing processes with realistic rates should be included in underlying hydrodynamic descriptions for quark-gluon plasma (QGP).

Because the energy density $e \sim \lambda T^{4}$, where $\lambda$ is the fugacity and $T$ the temperature, for keeping the energy density unchanged the temperature decreases by $20 \%$ when the fugacity increases from 0.5 to 1 . Thus, an effect of chemical equilibration is expected to be present for the temperature, which is an important detail, for instance for the dilepton [11, 15-17] and photon [18] yields in ultrarelativistic heavy-ion collisions. Moreover, to what degree chemical equilibration of quarks and gluons is achieved at the phase transition might also be crucial for modelling hadronization using recombination models [19].

In this work we develop a dissipative hydrodynamic approach including particle number changing processes via a rate equation to investigate the bulk properties of the QGP at RHIC. As a first step we simplify the expansion dynamics at RHIC by assuming a onedimensional expansion in the beam axis with Bjorken boost invariance. The QGP is considered to possess a constant $\eta / s$ value during its evolution. Different from the previous studies of chemical equilibration of the QGP basing on either ideal [10, 11] or first-order [20] hydrodynamic equations, we apply the second-order Israel-Stewart [9, 21] as well as the 
extended third-order hydrodynamic equations [22] to describe the QGP evolution. The hydrodynamic equations are now coupled with a rate equation for the particle number density. The microscopic interactions, which are responsible for the viscous behaviour and change of the particle number, are simplified by elastic binary $2 \rightarrow 2$ and inelastic multiplication and annihilation $2 \leftrightarrow 3$ processes with isotropically distributed collision angles. Although the evolution of multi-component systems as discussed in Ref. [23] is gaining an increasing attention, here we consider only a pure gluon system for the sake of simplicity.

To examine the applicability of the new description we compare the hydrodynamic solutions with those calculated from a parton cascade, the Boltzmann approach of multiparton scatterings (BAMPS) [13], in a similar manner as done in Refs. [9, 21, 22, 24, 25]. The $\eta / s$ is extracted from the BAMPS calculations using the procedure introduced by us in Ref. [21].

The paper is organised as follows. In Sec. II we introduce the dissipative hydrodynamic equations for a one-dimensional boost-invariant expanding system of gluons in presence of particle production and annihilation processes. Solutions are compared with those without the inelastic processes and those assuming chemical equilibrium. This quantitatively demonstrates the effects of chemical equilibration on physical observables. Influences of the initial conditions on these observables is studied in Sec. [II]. In Sec. IV] we compare the results of hydrodynamic calculations with those from BAMPS. Conclusions are given in Sec. V]

\section{VISCOUS HYDRODYNAMIC EQUATIONS AND RATE EQUATION DESCRIBING CHEMICAL EQUILIBRATION}

Derivations of dissipative hydrodynamic equations either from the entropy production principle or the moments method were reported in Refs. [22, 26, 27]. Ignoring the bulk viscosity and the heat conductivity, one obtains the evolution equation for the shear tensor

$$
\underbrace{\dot{\pi}^{\alpha \beta}=-\frac{\pi^{\alpha \beta}}{\tau_{\pi}}+\frac{\sigma^{\alpha \beta}}{\beta_{2}}-\pi^{\alpha \beta} \frac{T}{\beta_{2}} \partial_{\mu}\left(\frac{\beta_{2}}{2 T} u^{\mu}\right)}_{\text {IS }}+\underbrace{\alpha \frac{T}{\beta_{2}} \partial_{\mu}\left(\frac{\beta_{2}^{2}}{T} u^{\mu}\right) \pi_{\sigma}^{\langle\alpha} \pi^{\sigma \beta\rangle}}_{\text {third-order }},
$$

where

$$
\sigma^{\alpha \beta}=\nabla^{\langle\alpha} u^{\beta\rangle}=\left(\frac{1}{2}\left(\Delta_{\mu}^{\alpha} \Delta_{\nu}^{\beta}+\Delta_{\mu}^{\beta} \Delta_{\nu}^{\alpha}\right)-\frac{1}{3} \Delta_{\mu \nu} \Delta^{\alpha \beta}\right) \nabla^{\mu} u^{\nu}
$$

and $\Delta_{\alpha \beta}=g_{\alpha \beta}-u_{\alpha} u_{\beta}$ with the metric $g_{\alpha \beta}=\operatorname{diag}(1,-1,-1,-1) . u^{\mu}$ is the fluid velocity with $u_{\mu} u^{\mu}=1$. $\tau_{\pi}=2 \eta \beta_{2}$ is the relaxation time, where $\eta$ denotes the shear viscosity and 
$\beta_{2}=9 /(4 e)$. The "IS" part is exactly the equation from the Israel-Stewart theory, while the "third-order" part indicates a higher order correction term derived in Ref. [22]. We obtained $\alpha=-8 / 9$.

We simplify the dynamical evolution of a QGP created in ultrarelativistic heavy-ion collisions by a one-dimensional boost-invariant expansion of massless Boltzmann particles. For this case $u^{\mu}=(t, 0,0, z) / \tau$ where $\tau=\sqrt{t^{2}-z^{2}}$ is the proper time. With $\partial_{\mu} u^{\mu}=1 / \tau$ and $u^{\mu} \partial_{\mu}=\frac{d}{d \tau}$ Eq. (1) reduces to

$$
\dot{\bar{\pi}}=-\frac{\bar{\pi}}{\tau_{\pi}}+\frac{8}{27} \frac{e}{\tau}-\frac{1}{2} \frac{\bar{\pi}}{\tau}+\frac{1}{2} \bar{\pi} \frac{\dot{T}}{T}+\frac{1}{2} \bar{\pi} \frac{\dot{e}}{e}+\frac{3}{2} \frac{\bar{\pi}^{2}}{e \tau}+\frac{3}{2} \frac{\bar{\pi}^{2}}{e} \frac{\dot{T}}{T}+\frac{3}{2} \frac{\bar{\pi}^{2}}{e} \frac{\dot{e}}{e}-4 \frac{\bar{\pi}^{2}}{e \tau},
$$

where $\bar{\pi}=\pi^{33}=-2 \pi^{11}=-2 \pi^{22}$ is the only independent component of $\pi^{\mu \nu}$ in the local rest frame. The dot denotes the derivative with respect to $\tau$. Time evolution equation for the energy density $e$ follows from the conservation of energy, $\partial_{\mu} T^{\mu 0}=0$, where $T^{\mu \nu}$ is the energy-momentum tensor. For a one-dimensional boost-invariant system one obtains [22, 27]

$$
\dot{e}=-\frac{4}{3} \frac{e}{\tau}+\frac{\bar{\pi}}{\tau}
$$

Equation (3) corresponds to the Grad's ansatz [22] for an off-equilibrium distribution of particles in phase space

$$
f(x, p)=f_{e q}(x, p) \lambda\left[1+\frac{3 \bar{\pi}}{8 e T^{2}}\left(\frac{1}{2} p_{T}^{2}-p_{z}^{2}\right)\right],
$$

where $f_{e q}(x, p)=g e^{-E / T}$ is the equilibrium distribution. $g$ is the degeneracy factor and $g=$ 16 for gluons. $\lambda$ denotes the fugacity and quantifies the degree of the chemical equilibration. The distribution (5) satisfies the matching of the particle number and energy densities, $n=\lambda n_{e q}=\lambda g T^{3} / \pi^{2}$ and $e=\lambda e_{e q}=3 \lambda g T^{4} / \pi^{2}$, by which an off-equilibrium state is matched to a fictitious equilibrium state [28]. The matching conditions allow to define temperature for an off-equilibrium system. Assuming chemical equilibrium, i.e., $\lambda=1$, one obtains

$$
T=\left(\frac{\pi^{2} e}{3 g}\right)^{1 / 4}
$$

With this one can solve the coupled equations (3) and (4) once the initial conditions are given. The assumption of chemical equilibrium is made in most of the hydrodynamic calculations [2 4, 7, 8]. However, an instantaneous and complete chemical equilibrium requires an infinite transition rate of particle number changing processes, which is not consistent with a finite 
shear viscosity. Without the assumption of the chemical equilibrium, the temperature

$$
T=\frac{e}{3 n}
$$

depends also on the particle number density. Thus, we need an additional equation for the time evolution of $n$.

We assume that the space-time evolution of gluons obeys the Boltzmann equation

$$
p^{\mu} \partial_{\mu} f(x, p)=C[f]
$$

where $C[f]$ is the collision integral and contains microscopic details of particle interactions. Comparisons between the solutions of the hydrodynamic equations and those of the Boltzmann equation will be shown in Sec. IV. Here we use the Boltzmann equation to derive an equation for the particle number density $n$.

Integration of Eq. (8) over $d \Gamma=d^{3} p /\left[(2 \pi)^{3} E\right]$ leads to

$$
\int d \Gamma p^{\mu} \partial_{\mu} f(x, p)=\int d \Gamma C[f]
$$

The left hand side can be rewritten as the derivative of the particle number current, $\partial_{\mu} N^{\mu}$, which is equal to $\dot{n}+n / \tau$ for a one-dimensional boost-invariant expansion. The right hand side presents the source of particle production and annihilation. Considering two to three particles and vice versa as the only particle number changing processes, one obtains the rate equation [10, 11, 20, 29]

$$
\dot{n}+\frac{n}{\tau}=\frac{1}{2} n R_{23}-\frac{1}{3} n R_{32},
$$

where $R_{23}$ and $R_{32}$ denote the collision rates per particle for inelastic $2 \rightarrow 3$ and $3 \rightarrow 2$ processes. The factor $1 / 2$ and $1 / 3$ indicate that colliding particles are identical. The rates are given by [30, 31]

$$
R_{23}=n\left\langle v_{\mathrm{rel}} \sigma_{23}\right\rangle_{2}, \quad R_{32}=\frac{1}{2} n^{2}\left\langle\frac{I_{32}}{8 E_{1} E_{2} E_{3}}\right\rangle_{3}
$$

where the averages are defined as

$$
\begin{aligned}
\langle\mathcal{Q}\rangle_{2} & =\frac{1}{n^{2}} \int \frac{d^{3} p_{1}}{(2 \pi)^{3}} \frac{d^{3} p_{2}}{(2 \pi)^{3}} f\left(x, p_{1}\right) f\left(x, p_{2}\right) \mathcal{Q} \\
\langle\mathcal{Q}\rangle_{3} & =\frac{1}{n^{3}} \int \frac{d^{3} p_{1}}{(2 \pi)^{3}} \frac{d^{3} p_{2}}{(2 \pi)^{3}} \frac{d^{3} p_{3}}{(2 \pi)^{3}} f\left(x, p_{1}\right) f\left(x, p_{2}\right) f\left(x, p_{3}\right) \mathcal{Q} .
\end{aligned}
$$

$v_{\text {rel }}=\left(p_{1}+p_{2}\right)^{2} /\left(2 E_{1} E_{2}\right)$ is the relative velocity of two colliding particles with four momenta $p_{1}$ and $p_{2}$. The general definitions of the cross section $\sigma_{23}$ and $I_{32}$ can be found in Refs. 
[13, 31]. For interactions with isotropic distributions of collision angles $I_{32}$ is related to $\sigma_{23}$ via $I_{32}=192 \pi^{2} \sigma_{23}[13]$. Using the approximate distribution (5) we obtain

$$
\frac{1}{2} n R_{23}-\frac{1}{3} n R_{32}=\frac{1}{2} n^{2}(1-\lambda) \sigma_{23}
$$

which leads to

$$
\dot{n}+\frac{n}{\tau}=\frac{1}{2} n^{2}(1-\lambda) \sigma_{23} .
$$

Equation (15) is similar to the rate equations derived in Ref. [10].

The hydrodynamic equations (3), (4), and (15) describe kinetic and chemical equilibration of a gluon matter. Whereas the value of $\eta$ does not depend on the types of microscopic interactions [30], the net particle production, which explicitly affects the shear pressure $\bar{\pi}$ and $n$, is of course strongly dependent on the strength of inelastic collisions.

The standard viscous hydrodynamic approaches like the ones employed in Refs. [3, 4, 7] are up to second order in gradients and dissipative quantities. In the one-dimensional case studied here, the relevant gradient is the expansion scalar $\partial_{\mu} u^{\mu}=1 / \tau$ and the relevant dissipative quantity is the shear pressure $\bar{\pi}$. Both $\tau_{\pi} / \tau$ and $\bar{\pi} / e$ must be small to ensure the validity of the hydrodynamic approach. In our notation a term of $q$-th order has the form $\left(\tau_{\pi} / \tau\right)^{q_{1}}(\bar{\pi} / e)^{q_{2}}$ with $q_{1}+q_{2}=q$. To make a proper order counting for Eq. (3) we rewrite $\dot{T} / T=\dot{e} / e-\dot{n} / n$ according to Eq. (7) and insert Eqs. (41) and (15) for $\dot{e}$ and $\dot{n}$ into (3). We then multiply Eq. (3) by $\tau_{\pi} / e$. Keeping terms up to second order we obtain

$$
\dot{\bar{\pi}}=-\frac{\bar{\pi}}{\tau_{\pi}}-\frac{4}{3} \frac{\bar{\pi}}{\tau}+\frac{8}{27} \frac{e}{\tau}-\frac{1}{4} \bar{\pi} n(1-\lambda) \sigma_{23} .
$$

We refer to Eq. (16) as the Israel-Stewart (IS) equation. A similar equation, used in Refs. [9, 29] assuming particle number conservation, i.e., $\sigma_{23}=0$, contains the term $\bar{\pi}^{2} /(e \tau)$ which is neglected here, because this term times $\tau_{\pi} / e$ is of third order. Keeping terms up to third order we obtain

$$
\dot{\bar{\pi}}=-\frac{\bar{\pi}}{\tau_{\pi}}-\frac{4}{3} \frac{\bar{\pi}}{\tau}+\frac{8}{27} \frac{e}{\tau}-3 \frac{\bar{\pi}^{2}}{e \tau}-\frac{1}{4}\left(1+3 \frac{\bar{\pi}}{e}\right) \bar{\pi} n(1-\lambda) \sigma_{23} .
$$

We refer to Eq. (17) as the "third-order" equation.

In the following we solve the IS equation (16) as well as the third-order equation (17) coupled with Eqs. (4) and (15). We consider a gluon system with a constant $\eta / s=0.35$, which is a rough estimate of the upper bound of the $\eta / s$ value found at RHIC [4]. The 
entropy density $s$ is taken at kinetic equilibrium, i.e., at $\bar{\pi}=0$

$$
s=4 n-n \ln \lambda .
$$

The fugacity is related to $e$ and $n$ via $\lambda=n / n_{e q}=\left(\pi^{2} / g\right) n T^{-3}=\left(\pi^{2} / g\right) n(e / 3 n)^{-3}$. Since $\eta \sim e \lambda_{m f p}$, where $\lambda_{m f p}$ is the gluon mean free path, we have $\eta / s \sim(T / s)\left(n \lambda_{m f p}\right)$. With the choice

$$
\sigma_{23}=\frac{g}{\pi^{2}} \frac{T}{s}=\frac{1}{\lambda(4-\ln \lambda) T^{2}}
$$

and the same cross section of binary collisions $\sigma_{22}=\sigma_{23}$, a constant $\eta / s=0.35$ can be obtained in kinetic transport calculations, as will be shown in Fig 4 in Sec. IV.

To demonstrate effects of the chemical equilibration on observables we compare results of calculations with non-vanishing $\sigma_{23}$ to those with $\sigma_{23}=0$ (particle number conservation) and with $\lambda=1$ (instantaneous chemical equilibrium). In the case of $\sigma_{23}=0$ Eq. (15) gives $\dot{n}=-n / \tau$ and leads to $n(\tau)=n\left(\tau_{0}\right) \tau_{0} / \tau$. For $\lambda=1$ we have $n=n_{e q}=\left(3 g / \pi^{2}\right)^{1 / 4} e^{3 / 4} / 3$, which is the solution of Eq. (15) only if $\bar{\pi}=0$, i.e., $\eta=0$. This corresponds to infinite transition rates of particle number changing processes, which keep the system in chemical equilibrium. Here we also see that the setup of $\lambda=1$ and a non-vanishing shear viscosity $\eta$ is physically inconsistent.

Figure 1 shows the results for a thermal initial condition at $\tau_{0}=0.4 \mathrm{fm} / \mathrm{c}$ with $T\left(\tau_{0}\right)=0.5$ $\mathrm{GeV}$ and $\lambda\left(\tau_{0}\right)=1$. To see the effects of the finite shear viscosity and the particle number changing processes, we rescale the particle number density, the temperature, the energy and the entropy density by their time evolution in an ideal fluid. We first compare the results for non-vanishing $\sigma_{23}$ (solid curves), $\sigma_{23}=0$ (dashed curves) and $\lambda=1$ (dotted curves) using the IS equations. With the particle number conservation $\left(\sigma_{23}=0\right) n \cdot \tau / \tau_{0}=n\left(\tau_{0}\right)$ is constant. The non-vanishing $\sigma_{23}$ leads to a net increase of particle number, because the finite shear viscosity brings the system away from chemical equilibrium and the system becomes undersaturated $(\lambda<1)$. This is clearly demonstrated in the time evolution of the fugacity in Fig. 1 (b). It follows from Eq. (15) that the larger the value of $\sigma_{23}$, the larger is the particle number increase and thus the faster is the restoration of the chemical equilibrium. In the limit of instantaneous restoration $(\lambda=1)$, corresponding to $\sigma_{23} \rightarrow \infty$, a maximum of particle productions is achieved, as shown by the dotted curve in Fig. 11 (a).

Due to the viscous effect, the decrease of the energy density is slower than in the ideal fluid. Three curves in Fig. 1 (c) differ only marginally. Same is observed for the $\bar{\pi} / e$ ratio 

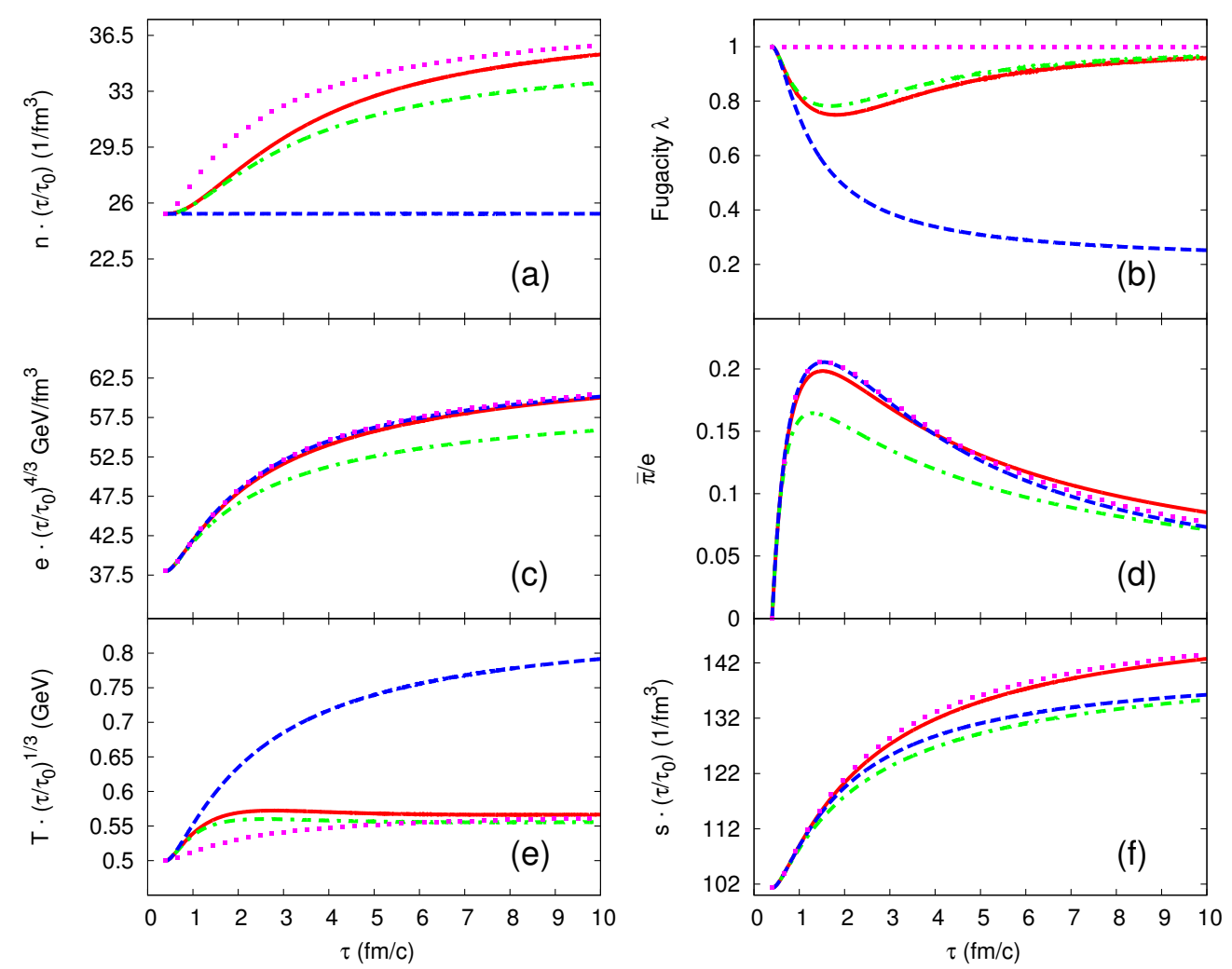

$\begin{array}{ll}\text { IS, } \eta / \mathrm{s}=0.35, \sigma_{23}=\left(\lambda(4-\ln \lambda) \mathrm{T}^{2}\right)^{-1}- & \text { IS, } \eta / \mathrm{s}=0.35, \lambda=1 \\ \text { IS, } \eta / \mathrm{s}=0.35, \sigma_{23}=0 & 3^{\text {rd }} \mathrm{O}, \eta / \mathrm{s}=0.35, \sigma_{23}=\left(\lambda(4-\ln \lambda) T^{2}\right)^{-1} \cdots\end{array}$

FIG. 1: (Color online) Time evolution of (a) the rescaled particle number density $n \cdot\left(\tau / \tau_{0}\right)$, (b) the fugacity $\lambda$, (c) the rescaled energy density $e \cdot\left(\tau / \tau_{0}\right)^{4 / 3}$, (d) the ratio $\bar{\pi} / e$, (e) the rescaled temperature $T \cdot\left(\tau / \tau_{0}\right)^{1 / 3}$ and (f) the rescaled entropy density $s \cdot\left(\tau / \tau_{0}\right)$. The cases $\sigma_{23}=0$ (dashed curves) and $\lambda=1$ (dotted curves) are calculated using the IS equations. The standard case with Eq. (19) is calculated using both the IS (solid curves) and the third-order equations (dash-dotted curves).

in Fig. 1 (d). Time evolution of the energy density and the shear pressure depend mainly on the viscosity and not much on the details of microscopic interactions. Formally, the weak dependence of $e$ and $\bar{\pi}$ on $\sigma_{23}$ or $\lambda$ is explained by the fact that the corresponding term in Eq. (3) is proportional to $\dot{T} / T$, which has a logarithmic dependence on the temperature.

Because of $T=e /(3 n)$ the rates of the particle number changing processes influence the value of the temperature. The larger the rates, which are infinity in the case of the instantaneous equilibration $(\lambda=1)$, the smaller is the temperature, as shown in Fig. 1(e). Between the two limits, $\sigma_{23}=0$ and $\lambda=1$, there is a $50 \%$ difference at the final time $\tau=10$ 
$\mathrm{fm} / \mathrm{c}$.

Due to the matching $n=\lambda n_{e q}$ and $e=\lambda e_{e q}$ we obtain $n \sim e^{3 / 4} \lambda^{1 / 4}$ and thus $s \sim$ $e^{3 / 4} \lambda^{1 / 4}(4-\ln \lambda)$ according to Eq. (18). Because the energy density is rather insensitive to the evolution of fugacity $\lambda$ [Fig. [1 (c)] and the function $\lambda^{1 / 4}(4-\ln \lambda)$ is almost constant for not very small $\lambda$, the entropy density weakly depends on the fugacity, see Fig. 1 (f). A larger effect can be observed at late times in the case of $\sigma_{23}=0$, because at that time the fugacity is quite small if particle number is conserved.

The third-order correction terms to the IS equations reduce the entropy production. This is the main finding of Ref. [22]. In Fig. 1](f) we observe that the entropy density calculated from the third-order equations (dash-dotted curve) is considerably smaller than that from the IS equations (solid curve). Thus, in oder to obtain the same $\eta / s$, a smaller shear viscosity $\eta$ is needed, which leads to smaller energy density and shear pressure, compared with the results of the IS equations. However, the interplay between these quantities and $n, T$, and $\lambda$ appears to be non-trivial.

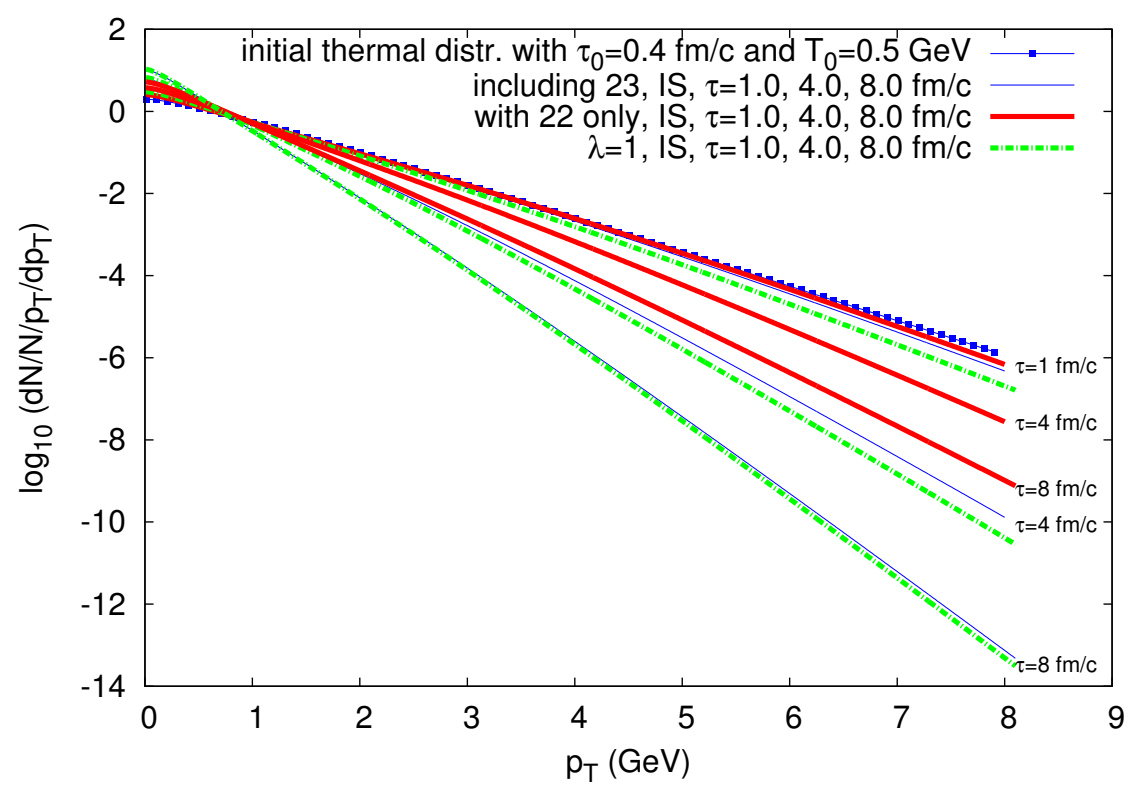

FIG. 2: (Color online) Transverse momentum spectra according to the distribution (5)). Depicted are the initial spectrum at $\tau_{0}=0.4 \mathrm{fm} / \mathrm{c}$ and spectra at $\tau=1 \mathrm{fm} / \mathrm{c}, 4 \mathrm{fm} / \mathrm{c}$ and $8 \mathrm{fm} / \mathrm{c}$.

Since the rates of the particle number changing processes influence the temperature, they should as well influence the particle momentum spectrum, which is more closely related to experimental data. Using the distribution (15) we calculate the particle transverse momentum 
spectra at different times and demonstrate them in Fig. 2. Values of $e, \bar{\pi}, T$, and $\lambda$ are taken from solutions of the IS equations in the three cases shown in Fig. 1, According to Eq. (5) the spectrum slope is approximately proportional to the temperature regardless of the dissipative correction. Significant differences in the spectra for the three cases at a late time, $\tau=4 \mathrm{fm} / \mathrm{c}$ or $\tau=8 \mathrm{fm} / \mathrm{c}$, reflect the differences in temperature shown in Fig. 1 (e).

\section{INFLUENCE OF INITIAL CONDITIONS}

In ultrarelativistic heavy-ion collisions initially produced quarks are much less abundand than gluons. Chemical equilibration of quarks and gluons start with different initial values of fugacity. The initial fugacity of quarks is expected to be much smaller than one [10]. In this section we repeat calculations performed for Fig. 1 but with an initial fugacity $\lambda\left(\tau_{0}\right)=0.2$ instead of 1 . The initial condition is a kinetically equilibrated, but chemically disequilibrated system. The initial energy density is $e\left(\tau_{0}\right)=\lambda\left(\tau_{0}\right) e_{e q}\left(\tau_{0}\right)=\left(3 g / \pi^{2}\right) \lambda\left(\tau_{0}\right)\left[T\left(\tau_{0}\right)\right]^{4}$ with $T\left(\tau_{0}\right)=0.5 /\left[\lambda\left(\tau_{0}\right)\right]^{1 / 4} \mathrm{GeV}$. We calculate the IS equations, Eqs. (16)), (41), and (15), for $\eta / s=0.35$.

The results are shown in Fig. 3 for three cases: non-vanishing $\sigma_{23}$ [Eq. (19)] by the solid curves, $\sigma_{23}=0$ by the dashed curves, and $\lambda=1$ by the dotted curves. In the case of $\lambda=1$ the initial temperature is $0.5 \mathrm{GeV}$; it is larger than $0.5 \mathrm{GeV}$ in case $\lambda_{0}=0.2$ to hold the same initial energy density. The initial particle number density and the entropy density are larger in the $\lambda=1$ case compared with the values of the other two cases.

As already observed in Fig. 1, the energy density and the shear pressure, shown in Fig. 3 (c) and (d), are weakly affected by the value of $\sigma_{23}$. On the contrary, without particle number changing processes, $\sigma_{23}=0$, the fugacity [see Fig 3 (b)] decreases and the system goes far away from the chemical equilibrium, while the fugacity increases to 1 for large $\sigma_{23}$. Accordingly, pronounced differences are seen in the particle number density [Fig. 3 (a)], the temperature [Fig. 3 (e)], and the entropy density [Fig. 3 (f)] between the non-vanishing $\sigma_{23}$ and $\sigma_{23}=0$ cases. We also observe that the results of $n, \lambda$ and $T$ in the non-vanishing $\sigma_{23}$ case relax to those of the $\lambda=1$ case.

Comparisons in Fig. 3 underline the importance of microscopic details in applying hydrodynamic approaches to describe both chemical and kinetic equilibration of an initially undersaturated system. 

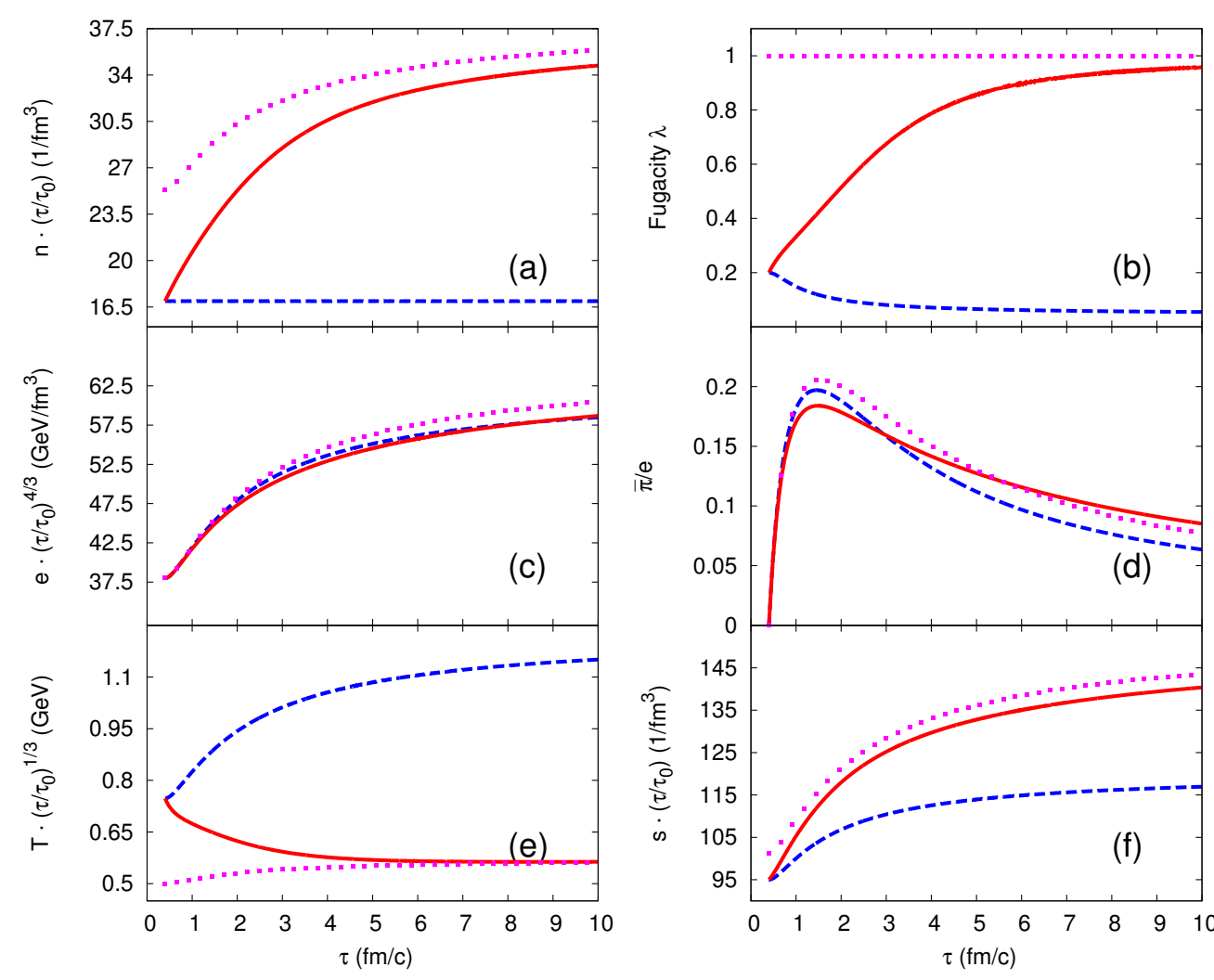

IS, $\eta / \mathrm{s}=0.35, \sigma_{23}=\left(\lambda(4-\ln \lambda) \mathrm{T}^{2}\right)^{-1}, \lambda_{0}=0.2$

IS, $\eta / \mathrm{s}=0.35, \sigma_{23}=0, \lambda_{0}=0.2$

$----$

IS, $\eta / s=0.35$, constant $\lambda=1 \cdots$

FIG. 3: (Color online) Same as Fig. 1 using the IS equation (16) with kinetically equilibrated and chemically disequilibrated initial condition, $\lambda\left(\tau_{0}\right)=0.2$.

\section{COMPARISONS BETWEEN THE HYDRODYNAMIC AND TRANSPORT CALCULATIONS}

Kinetic transport theory describes the space-time evolution of particles by means of detailed treatment of microscopic processes. The dynamical behaviour of a particle system resembles the one described by viscous hydrodynamics if the particle mean free path is much smaller than macroscopic scales. Comparisons between hydrodynamic and transport calculations are always useful to examine the applicability limits of viscous hydrodynamic theories [9, 21, 22, 24, 25].

In this section we compare results from the new viscous hydrodynamic description including particle number changing processes presented in Sec. II with results of transport calculations using the parton cascade BAMPS [13]. BAMPS simulates particle interactions, 
especially inelastic $2 \leftrightarrow 3$ processes with full detailed balance, by using the stochastic interpretation of transition rates.

From the kinetic theory point of view, the physical origin of a finite shear viscosity is the finite rates of microscopic collision processes. The relation between the (transport) collision rate and the shear viscosity coefficient was reported in Refs. [21, 32]. Here we use the formula derived in [21]

$$
\eta=-\frac{\pi_{\mu \nu} \pi^{\mu \nu}}{2 T C_{0} \pi_{\mu \nu} P^{\mu \nu}}
$$

with $P^{\mu \nu}=\int d \Gamma p^{\mu} p^{\nu} C[f]$ and $C_{0}=3 /\left(8 e T^{2}\right)$. The collision integral $C[f]$ contains details of microscopic interactions. We consider elastic binary $2 \rightarrow 2$ and inelastic $2 \leftrightarrow 3$ processes with isotropic distributions of collision angles. The cross sections are parametrized by

$$
\sigma_{22}=\sigma_{23}=\frac{k}{\lambda(4-\ln \lambda) T^{2}},
$$

where $k$ is a parameter controlling the value of $\eta$. With this choice one expects to obtain a constant $\eta / s$ as discussed around Eq. (19). The transition probabilities of a $2 \rightarrow 2$ or $2 \rightarrow 3$ process within a unit volume $\Delta v$ and a unit time step $\Delta t$ are same and given by $P_{23}=P_{22}=v_{r e l} \sigma_{23} \Delta t / \Delta v$. The transition probability of a $3 \rightarrow 2$ process is $P_{32}=$ $\left(1 / 8 E_{1} E_{2} E_{3}\right) I_{32} \Delta t / \Delta v^{2}$ with $I_{32}=192 \pi^{2} \sigma_{23}$ [13]. Figure 4 shows the $\eta / s$ ratio extracted from BAMPS calculations with $k=0.5,1,3$, and 6 via Eq. (20). $\eta / s$ decreases with increasing $k$. Each ratio is in good agreement with a constant. These constant $\eta / s$ values and corresponding $\sigma_{23}$ parametrizations are used for solving the hydrodynamic equations (16) (IS) and (17) (third-order) together with Eqs. (41) and (15).

Figures 5, 6, 7, 8, and 9 show the comparisons between the hydrodynamic and transport calculations for a one-dimensional boost-invariant expansion of a gluon system with the thermal initial condition $T\left(\tau_{0}\right)=0.5 \mathrm{GeV}, \lambda\left(\tau_{0}\right)=1$ at $\tau_{0}=0.4 \mathrm{fm} / \mathrm{c}$. The results are rescaled in the same manner as previously done in Fig. 1.

In Figs. 5 and $[6$ we observe that the energy density and the shear pressure from the third-order hydrodynamic calculations agree perfectly with the BAMPS results even for large $\eta / s$ values. In contrast, the viscous effect is overestimated in the IS equations. This becomes significant for large $\eta / s$ values, which mark the applicability boundary of the IS approach. These findings are in line with those in our previous work [22], where particle changing processes were not taken into account. 


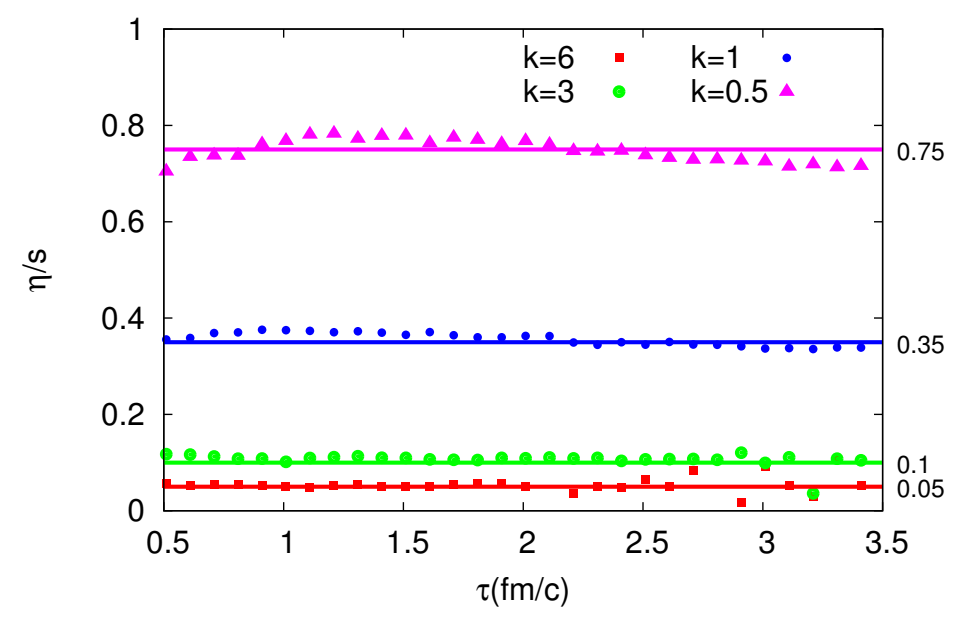

FIG. 4: (Color online) $\eta / s$ ratio extracted from BAMPS calculations with time-dependent cross sections.

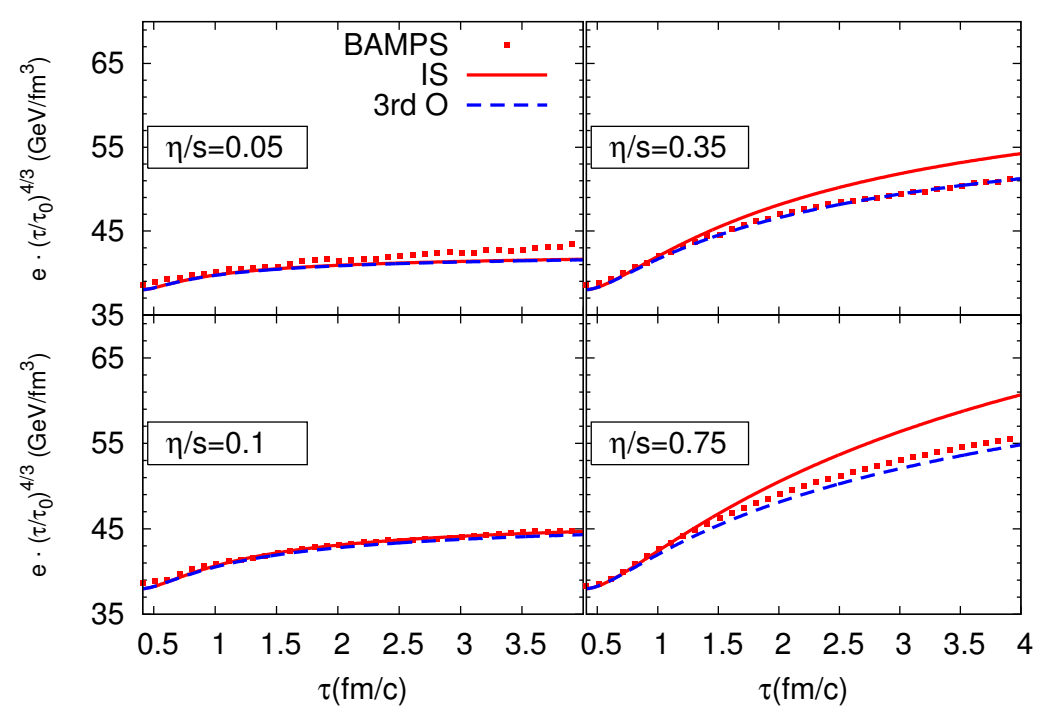

FIG. 5: (Color online) Rescaled energy density from BAMPS, IS and third-order hydrodynamic calculations.

The difference in the particle number density (Fig. 7) between the hydrodynamic and BAMPS results is large at large $\eta / s$ values. Although both IS and third-order equations give larger densities than those in BAMPS, the third-order results are closer to the ones from BAMPS . On the other hand, looking at the temperature $T$ (Fig. 8) and the fugacity $\lambda$ (Fig. 9) we observe that the IS results show better agreement with the BAMPS results than the third-order ones. However, it is difficult to make conclusions about applicability of 


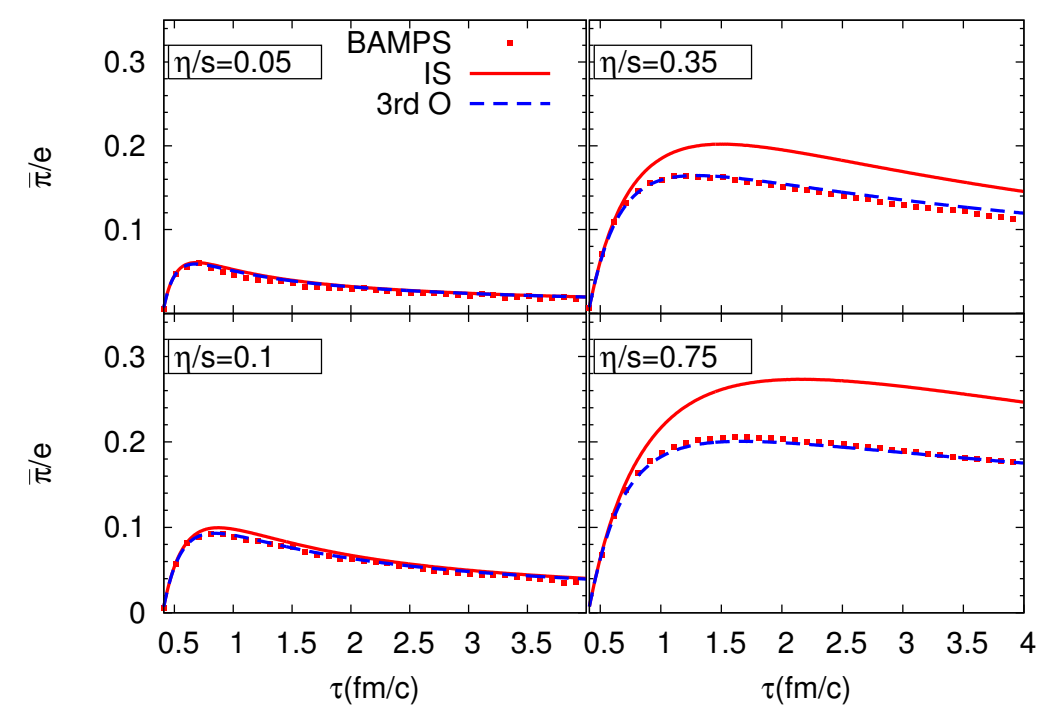

FIG. 6: (Color online) Shear pressure to energy density ratio from BAMPS, IS and third-order hydrodynamic calculations.

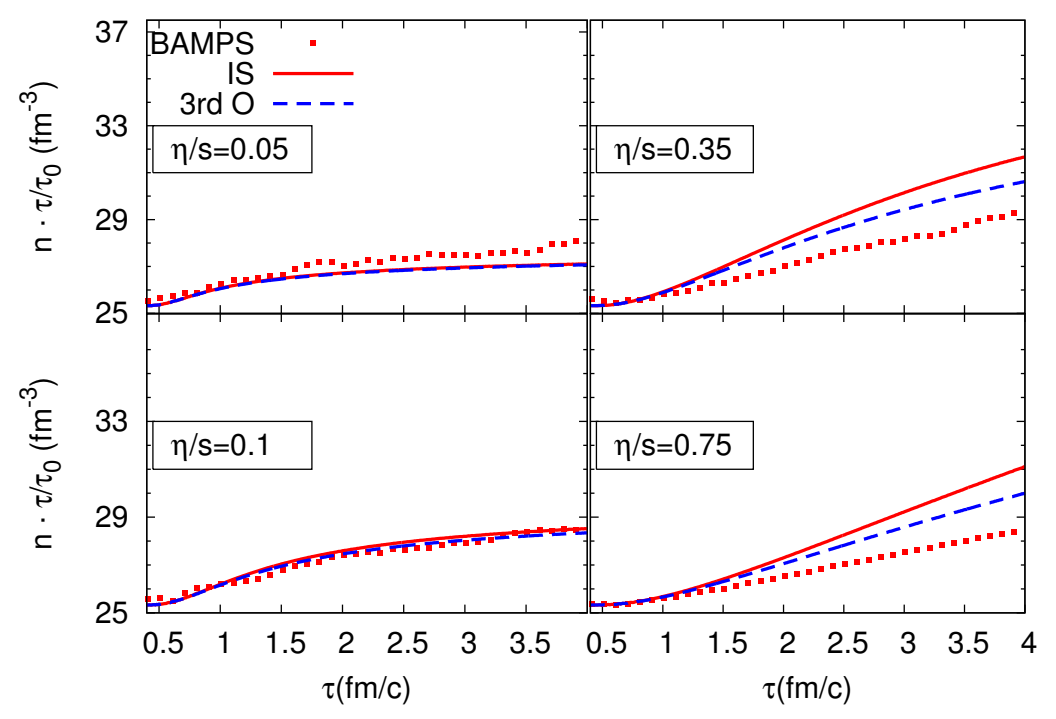

FIG. 7: (Color online) Rescaled particle number density from BAMPS, IS and third-order hydrodynamic calculations.

a hydrodynamic approach basing on the observables $T$ and $\lambda$. These quantities are defined via $e$ and $n$, but not solved directly from the hydrodynamic equations. If we consider the particle number conservation, the results on $T$ and $\lambda$ from the third-order calculations are in very good agreement with those from BAMPS, as can be cocluded from our observations in Ref. [22]. In the situation considered here, small differences between hydrodynamic and 


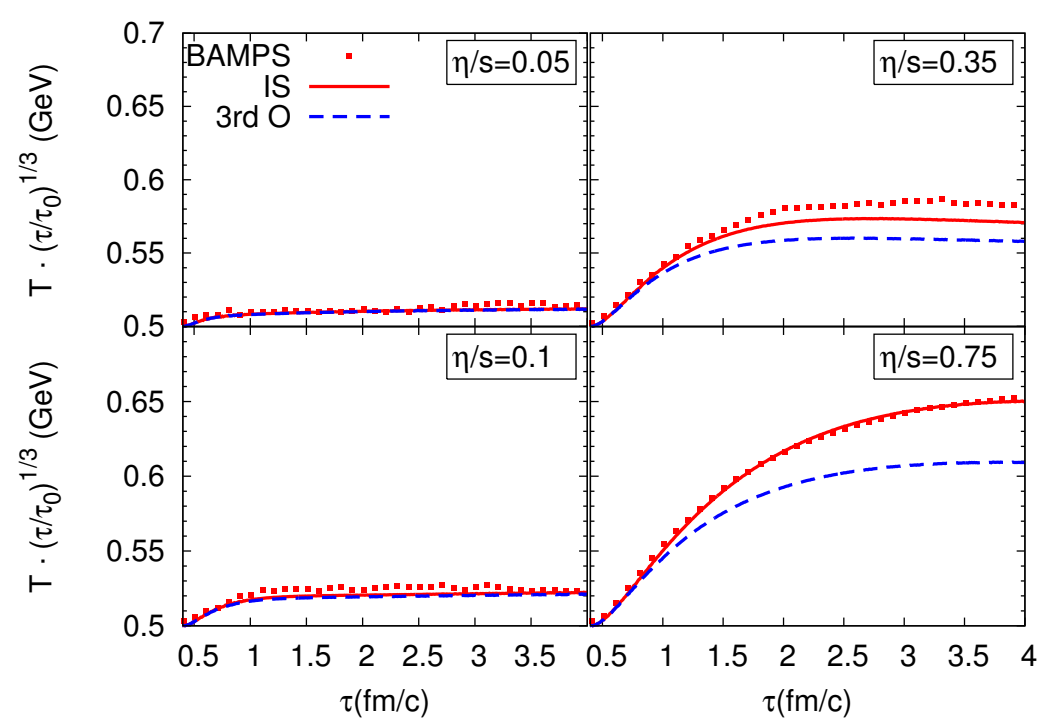

FIG. 8: (Color online) Rescaled effective temperature $T=e /(3 n)$ from BAMPS, IS and third-order hydrodynamic calculations.

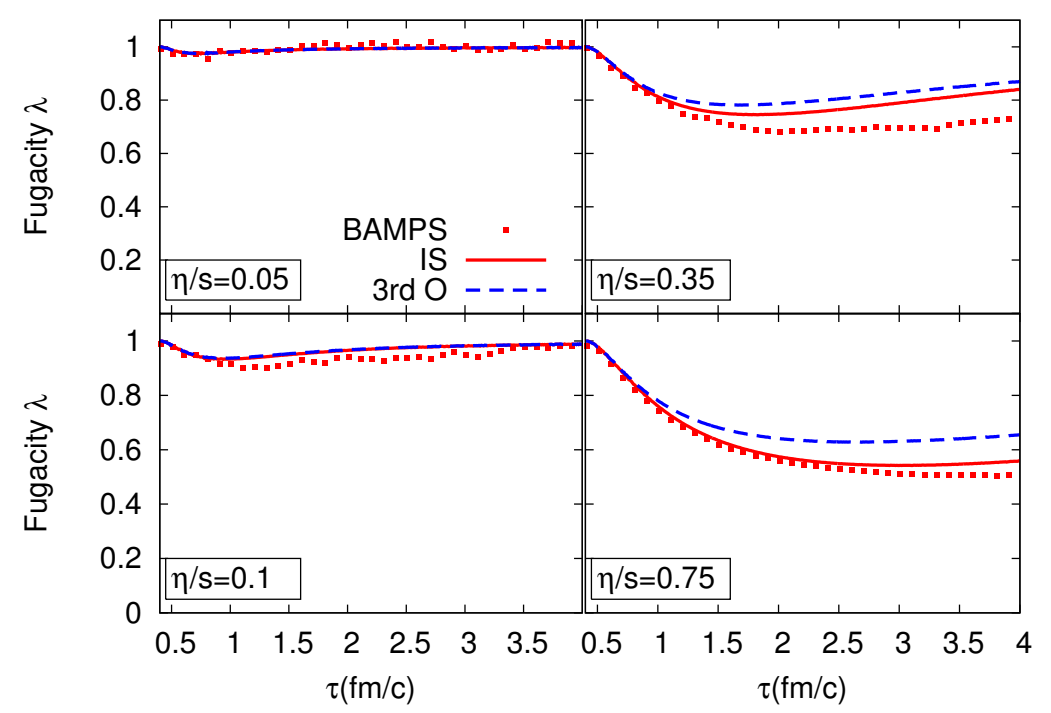

FIG. 9: (Color online) Fugacity from BAMPS, IS and third-order hydrodynamic calculations.

kinetic transport results on both key observables $e$ and $n$ translate into differences in $T$ and $\lambda$ in a non-trivial way.

To understand the differences in particle number densities between the hydrodynamic and BAMPS results, we examine Eq. (14), which is valid by virtue of the approximation (5). For this we calculate the left and the right hand sides of Eq. (14) using the actual values of $R_{23}$, $R_{32}, n, \lambda$, and $\sigma_{23}$ extracted from the BAMPS calculations. The results are shown in Fig. 


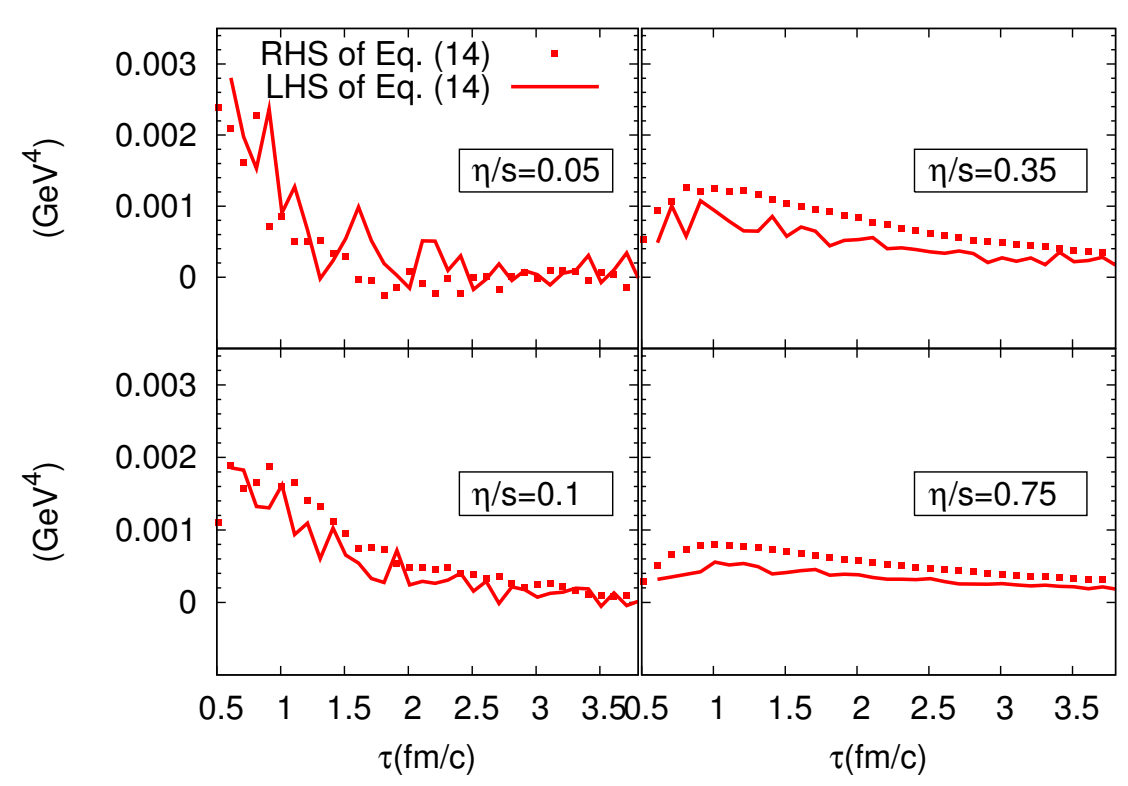

FIG. 10: (Color online) Left and right hand sides of Eq.(14) (denoted by LHS and RHS, respectively) calculated using the actual values of $R_{23}, R_{32}, n, \lambda$ and $\sigma_{23}$ from BAMPS.

10. Except for the case of $\eta / s=0.05, n^{2}(1-\lambda) \sigma_{23} / 2$ is always larger than $n R_{23} / 2-n R_{32} / 3$, which leads to a stronger particle production in the hydrodynamic than in the transport approach, as seen in Fig. 7. Therefore, it is obvious that the approximate distribution (5) must deviate from the one extracted from BAMPS. This deviation is demonstrated in Fig. 11 by the ratio of $p_{T}$ spectra at midrapidity extracted from BAMPS to the ones calculated using the Grad's approximation (5). The results are presented for $\eta / s=0.35$ at various times. The deviations of the ratios from 1 are on a level of $10 \%-20 \%$ and become more pronounced at later times, $\tau \sim 1-2 \mathrm{fm} / \mathrm{c}$, at which the viscous effect is strongest, see Fig. 6. We thus need a modification of the Grad's approximation, which is a highly interesting project for future studies.

\section{SUMMARY AND OUTLOOK}

In this work we have presented an extended set of viscous hydrodynamic equations, in which particle number changing processes are taken into account via a rate equation. The rate equation of the particle number density is derived from the Boltzmann equation employing the Grad's approximation. A one-dimensional boost-invariant expansion is considered 


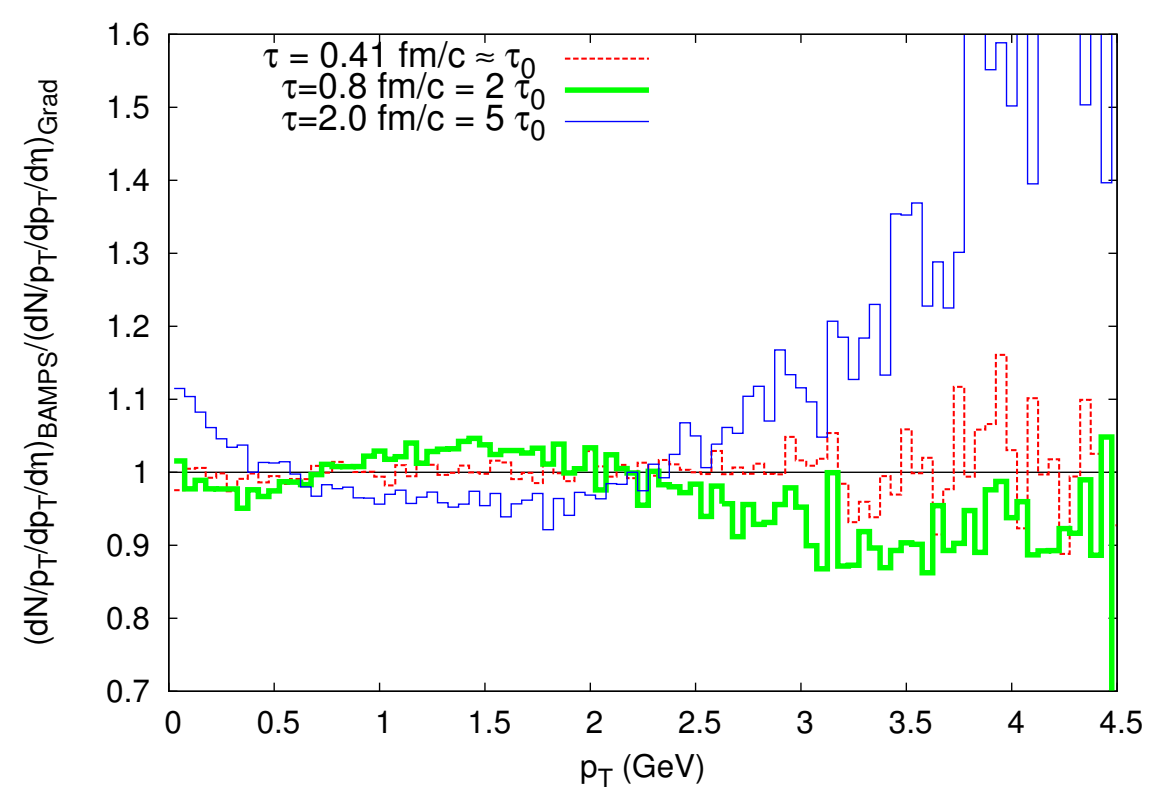

FIG. 11: (Color online) Ratio of transverse momentum spectrum extracted in BAMPS to the one calculated using the Grad's approximation at different times with $\eta / s=0.35$.

for simplifying the derivation of the hydrodynamic equations. We have demonstrated that a proper treatment of particle number evolution is essential for a hydrodynamic description of the gluon system, especially for describing chemical equilibration and for determining the temperature and the momentum spectra of the system.

We have compared the results between the hydrodynamic and transport calculations including inelastic $2 \leftrightarrow 3$ processes. The energy density and the shear pressure obtained from the third-order hydrodynamic equations agree well with the results from the transport approach using BAMPS even at large $\eta / s=0.75$, while the results from the Israel-Stewart hydrodynamics deviate from the BAMPS results by $10 \%-20 \%$ for $\eta / s=0.35-0.75$. Both the IS and the third-order hydrodynamic calculations fail to meet the BAMPS results on the particle number density at large $\eta / s$. The reason is that at large $\eta / s$ the viscous effect is so large that the Grad's ansatz for the off-equilibrium distribution should be modified beyond the second order in momentum, in order to give a more accurate rate equation of the particle number density. This is a highly interesting and timely subject for future investigations.

The parton cascade BAMPS is presently being extended by including light quark degrees of freedom [33]. Light quarks are expected to equilibrate both kinetically and chemically 
slower than a pure gluon system. Kinetic and chemical off-equilibrium effects will have significant influence on the jet and bulk medium physics. They will also be highly relevant concerning the slopes of transverse spectra and the yields of dileptons and photons, which were measured at RHIC [34] to extract the initial temperature for hydrodynamic approaches.

\section{Acknowledgements}

A. E. gratefully acknowledges fellowship by the Helmholtz foundation and thanks the University of Cape Town Physics Department for its hospitality during the stay in Cape Town, where this work was partly completed. A. M. acknowledges the support from the National Research Foundation through the NRF-DFG grant. The BAMPS simulations were performed at the Center for Scientific Computing of Goethe University. This work is supported by BMBF and by the Helmholtz International Center for FAIR within the framework of the LOEWE program (LandesOffensive zur Entwicklung Wissenschaftlichökonomischer Exzellenz) launched by the State of Hesse.

[1] S. A. Voloshin, A. M. Poskanzer and R. Snellings, arXiv:0809.2949 [nucl-ex].

[2] P. Romatschke and U. Romatschke, Phys. Rev. Lett. 99, 172301 (2007) arXiv:0706.1522 [nucl-th]].

[3] H. Song and U. W. Heinz, Phys. Rev. C 77, 064901 (2008) [arXiv:0712.3715 [nucl-th]].

[4] M. Luzum and P. Romatschke, Phys. Rev. C 78, 034915 (2008) [Erratum-ibid. C 79, 039903 (2009)] [arXiv:0804.4015 [nucl-th]].

[5] U. W. Heinz, arXiv:0901.4355 [nucl-th].

[6] D. A. Teaney, arXiv:0905.2433 [nucl-th].

[7] E. Molnar, Eur. Phys. J. C 60, 413 (2009) [arXiv:0807.0544 [nucl-th]].

[8] E. Molnar, H. Niemi and D. H. Rischke, Eur. Phys. J. C 65, 615 (2010) arXiv:0907.2583 [nucl-th]].

[9] P. Huovinen, D. Molnar, Phys. Rev. C 79 (2009) 014906.

[10] T. S. Biro et al, Phys. Rev. C 48 (1993) 1275.

[11] D. K. Srivastava, M. G. Mustafa and B. Müller, Phys. Rev. C 56, 1064 (1997) 
arXiv:nucl-th/9611041.

[12] D. M. Elliott, D. H. Rischke, Nucl. Phys. A 671 (2000) 583

[13] Z. Xu and C. Greiner, Phys. Rev. C 71, 064901 (2005) arXiv:hep-ph/0406278.

[14] F. Gelis, K. Kajantie and T. Lappi, Phys. Rev. Lett. 96, 032304 (2006) arXiv:hep-ph/0508229.

[15] K. Dusling and S. Lin, Nucl. Phys. A 809, 246 (2008) [arXiv:0803.1262 [nucl-th]].

[16] M. Martinez and M. Strickland, Phys. Rev. C 78, 034917 (2008) arXiv:0805.4552 [hep-ph]].

[17] H. van Hees and R. Rapp, Nucl. Phys. A 827, 341C (2009) arXiv:0901.2316 [nucl-th]].

[18] B. Schenke and M. Strickland, Phys. Rev. D 76, 025023 (2007) arXiv:hep-ph/0611332.

[19] Z. w. Lin and C. M. Ko, Phys. Rev. Lett. 89, 202302 (2002) arXiv:nucl-th/0207014; V. Greco, C. M. Ko and P. Levai, Phys. Rev. Lett. 90, 202302 (2003) arXiv:nucl-th/0301093; Phys. Rev. C 68, 034904 (2003) arXiv:nucl-th/0305024; R. J. Fries, B. Müller, C. Nonaka and S. A. Bass, Phys. Rev. Lett. 90, 202303 (2003) arXiv:nucl-th/0301087; Phys. Rev. C 68, 044902 (2003) arXiv:nucl-th/0306027; D. Molnar and S. A. Voloshin, Phys. Rev. Lett. 91, 092301 (2003) arXiv:nucl-th/0302014; R. C. Hwa and C. B. Yang, Phys. Rev. C 67, 064902 (2003) arXiv:nucl-th/0302006.

[20] D. Dutta, A. K. Mohanty, K. Kumar and R. K. Choudhury, Phys. Rev. C 61, 034902 (2000) arXiv:hep-ph/9908359.

[21] A. El, A. Muronga, Z. Xu and C. Greiner, Phys. Rev. C 79, 044914 (2009) arXiv:0812.2762 [hep-ph]].

[22] A. El, Z. Xu and C. Greiner, Phys. Rev. C 81, 041901 (2010) arXiv:0907.4500 [hep-ph]].

[23] A. Monnai and T. Hirano, arXiv:1003.3087 [nucl-th].

[24] I. Bouras et al., Phys. Rev. Lett. 103, 032301 (2009) arXiv:0902.1927 [hep-ph]]; Nucl. Phys. A 830, 741C (2009) arXiv:0907.4519 [hep-ph]]; arXiv:1006.0387 [hep-ph]; I. Bouras et al., Acta Phys. Polon. B 40, 973 (2009).

[25] G. S. Denicol, T. Koide and D. H. Rischke, arXiv:1004.5013 [nucl-th].

[26] W. Israel, Ann. Phys. (N.Y.) 100 (1976) 310; J.M. Stewart, Proc. R. Soc. London, Ser. A 357 (1977) 59; W. Israel, M. Stewart, Ann. Phys. (N.Y.) 118 (1979) 341.

[27] A. Muronga, Phys. Rev. C 69 (2004) 034903.

[28] A. Muronga, Phys. Rev. C 76 (2007) 014910.

[29] A. Muronga, J. Phys. G 31 (2005) 1035-1039. 
[30] Z. Xu and C. Greiner, Phys. Rev. C 76, 024911 (2007) arXiv:hep-ph/0703233.

[31] Z. Xu and C. Greiner, Phys. Rev. C 81, 054901 (2010) [arXiv:1001.2912 [hep-ph]].

[32] Z. Xu, C. Greiner, Phys. Rev. Lett. 100 (2008) 172301.

[33] O. Fochler, Z. Xu, C. Greiner, in preparation.

[34] S. S. Adler et al. [PHENIX Collaboration], Phys. Rev. Lett. 94, 232301 (2005) arXiv:nucl-ex/0503003]; A. Adare et al. [PHENIX Collaboration], Phys. Rev. C 81, 034911 (2010) arXiv:0912.0244 [nucl-ex]]. 